\title{
Left ventricular dysfunction: causes, natural history, and hopes for reversal
}

\author{
Paul W Armstrong
}

Left ventricular dysfunction (LVD) with subsequent congestive heart failure (CHF) constitutes the final common pathway for a host of cardiac disorders. Coronary artery narrowing or ischaemic heart disease is the dominant cause of heart failure and is often associated with acute or prior myocardial infarction. The remaining aetiologies include cardiomyopathy, hypertension, and a variety of other factors such as valve disease or myocarditis.

Heart failure is an enormous problem. Data from the Framingham heart study shows that it develops in approximately $16 \%$ of men and $18 \%$ of women who have diabetes; $12 \%$ of men and $8 \%$ of women who have hypertension; and $30 \%$ of both sexes who have myocardial infarction. ${ }^{1}$ Interestingly, over the second half of the 20th century there has been a striking increase in the frequency of coronary artery disease and diabetes as aetiological factors for $\mathrm{CHF}$, whereas the impact of hypertension and rheumatic valve disease has declined.

\section{Heart structure and function}

LVD produces many changes in the structure and function of the heart through a variety of mechanisms.

The muscle of the heart is encased in a collagen weave. There are interstitial spaces that are associated with a variety of elements, a number of which can contribute to the development of CHF. The extracellular matrix has a scaffolding function, which supports myocytes and blood vessels. It also provides lateral connections between the cells and muscular bundles that govern not only the architecture of the heart, but also its ability to contract. Moreover, the extracellular matrix contributes to the heart's tensile strength and

Correspondence to: Professor Armstrong resilience, which helps resist deformation, maintaining the elliptical shape of the heart and its thickness.

\section{Ventricular remodelling}

Once left ventricular dysfunction occurs a series of compensatory mechanisms are triggered which lead to a host of structural and neurohormonal adaptations. Haemodynamic, neurohormonal, and molecular factors operate to modulate remodelling of the left ventricle and vascular tree (fig 1). Ventricular remodelling is the ability to reconstruct the heart as a result of myocardial damage, with changes in ventricular thickness and size. These apply to the subcellular, the cellular, the tissue, and the chamber levels of the heart.

Following myocardial infarction, one phenomenon that is known to occur is ventricular expansion. With expansion of the ventricle there is dilatation and thinning which can occur without additional necrosis. There is also a distortion in the shape of the heart from an elliptical to a more spherical form. This contributes to substantial mechanical inefficiency and worsening of CHF.

There is an important relation between the size of the heart and patient outcome, with a progressive rise in mortality as end systolic and end diastolic volume increase. ${ }^{2}$ Reperfusion is critically important for ventricular healing and reducing the risk of ventricular aneurysm, which is an important precursor of $\mathrm{CHF}$.

\section{Neurohormonal network and LVD}

A number of neurohormones may be triggered as a result of myocardial dysfunction (fig 2). These neurohormones have both vasodilator and vasoconstrictor effects and provide a number of therapeutic opportunities.

One of the key neuroendocrine axes is the renin-angiotensin system. The other major neurohormonal axis operational in heart failure is the sympathetic nervous system. The interaction between these two systems is complex.

\section{RENIN-ANGIOTENSIN SYSTEM}

The renin-angiotensin system is one of the major neuroendocrine axes involved in the development of heart failure. Inhibition of this system therefore has important therapeutic effects. For example, angiotensin converting enzyme (ACE) inhibitors have a dual mechanism. Firstly, they inhibit the conversion of angiotensin I to angiotensin II and therefore reduce vasoconstriction and cell proliferation. Secondly, they inhibit the breakdown and metabolism of bradykinin and

- Hepatic
- Skeletal muscle
- Other




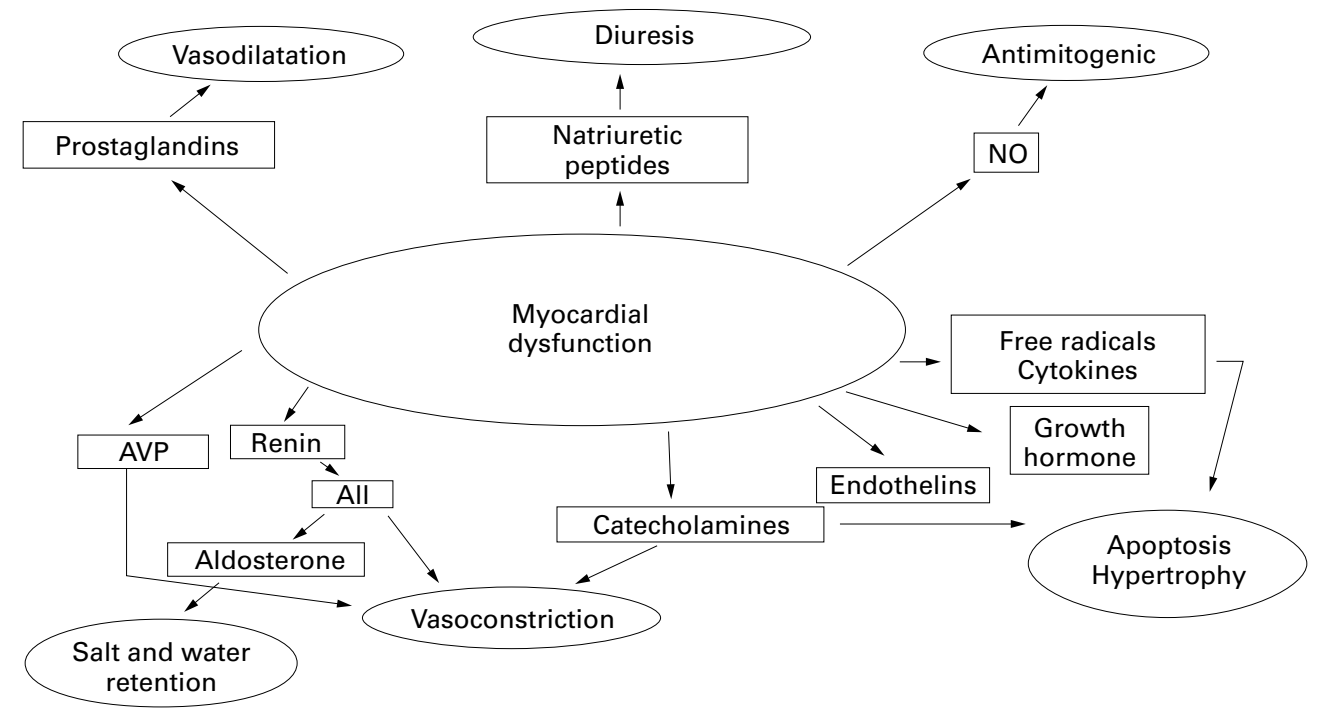

Figure 2 Many different neurohormonal pathways are stimulated following myocardial dysfunction.

therefore increase the production of prostaglandins and nitric oxide. The importance of this second mechanism is still being debated.

\section{ALDOSTERONE}

The emergence of aldosterone as an important player in CHF has recently been re-emphasised by survival benefits associated with spironolactone in the RALES study. ${ }^{3}$ Aldosterone is known to have multiple effects including sodium retention, potassium loss, increasing blood pressure, and baroreceptor dysfunction. In addition there is experimental evidence that spironolactone's antifibrotic effect may protect the matrix of the heart from unfavourable remodelling. ${ }^{4}$

\section{CATECHOLAMINES}

Catecholamines have a number of adverse effects related to heart failure. These include diminishing the ability of heart failure patients to respond to the demands of exercise by increasing both oxygen consumption and energy depletion. They are also involved in arrhythmogenesis, hypertrophy, alteration of the geometry of the heart, and potentiation of cell death and fibrosis.

\section{APOPTOSIS}

One important and recently appreciated phenomenon related to myocardial dysfunction is programmed cell death, or apoptosis, which may be important in the genesis and the worsening of CHF. Cardiac abnormalities may stimulate cytokines and growth factors, and with an increase in intracellular calcium and oxidative stress there may be apoptosis and progression of $\mathrm{CHF}$.

\section{Treatment}

THERAPEUTIC GOALS

The therapeutic goals in CHF are to enhance the quality of life of patients, improve survival, halt disease progression, and reverse the disease process. Above all, treatment should do no harm.
The ideal pharmacotherapy for heart failure would:

- reduce heart rate;

- reduce oxygen consumption;

- reduce neurohormonal activation;

- restore autonomic balance;

- enhance tissue and coronary perfusion;

- reduce circulatory congestion;

- promote favourable cardiac and vascular remodelling;

- restore cardiac size and shape.

\section{ACE INHIBITION}

The largest body of clinical trial evidence exists for the favourable benefits of ACE inhibitors in post-myocardial infarction or CHF patients with LVD. They have therefore become the standard of care for patients with LVD irrespective of symptoms.

In a study of patients soon after myocardial infarction, increases in diastolic and systolic volume occurred in patients receiving placebo. ${ }^{5}$ However, treatment with the ACE inhibitor captopril preserved diastolic and systolic volumes. This effect was maintained after withdrawal of treatment, suggesting that it was not drug dependent but had been associated with structural remodelling that persisted after withdrawal of the ACE inhibitor.

The SOLVD study found that, if patients had not suffered myocardial infarction or angina, they did much better than those who had. ${ }^{6}$ This emphasises the catastrophic impact of myocardial infarction in patients with established heart failure. Interestingly, ACE inhibitors have an independent effect on the reduction of myocardial infarction. Data from the SAVE study showed a reduction in the frequency of myocardial infarction with ACE inhibition (fig 3). ${ }^{7}$ The protective effects of ACE inhibitors therefore not only relate to their effects on the left ventricle but also on the coronary tree itself. In fact, a review of data from SOLVD, SAVE, and AIRE shows an overall $21 \%$ reduction in the risk of myocardial infarction in CHF patients when treated with ACE inhibitors. ${ }^{8}$ 


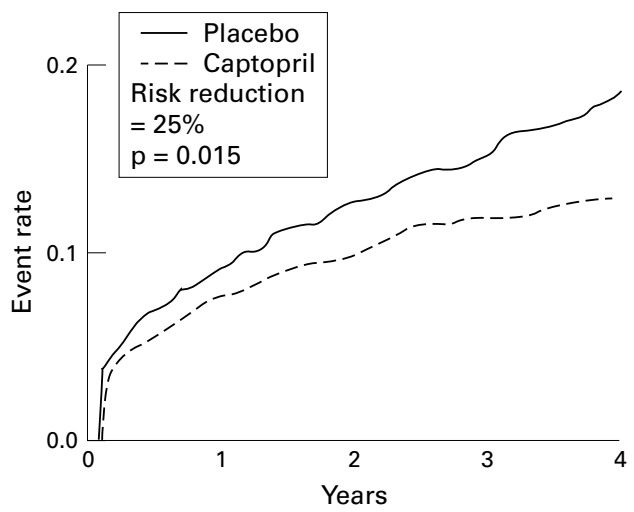

Figure 3 The SAVE study showed that captopril reduced the rate of myocardial infarction.

There are a number of mechanisms by which ACE inhibitors may reduce vascular events in patients with CHF:

- antihypertensive effect;

- anti-renin effect;

- enhancement of coronary endothelial function;

- antiproliferative effect;

- antiplatelet activity;

- modulation of fibrinolytic balance.

SPIRONOLACTONE

In the RALES study 1600 patients with New York Heart Assocation (NYHA) functional class III and IV heart failure, who had diminished ejection fractions and were receiving diuretics and, for the most part, ACE inhibitors, were given spironolactone in doses of $25 \mathrm{mg}$ to $50 \mathrm{mg}$ daily. ${ }^{3}$ The study was terminated early because of a striking reduction in all cause mortality of $27 \%$, and a commensurate reduction in cardiovascular hospitalisations with spironolactone. For 1000 patients treated for two years, there were 72 lives saved and 264 hospitalisations prevented. The introduction of spironolactone is therefore an important addition to the therapeutic armamentarium for CHF.

$\beta$ BLOCKERS

Recently new and compelling evidence from a number of sources supports the use of $\beta$ blocker treatment. $\beta$ Blockers provide incremental benefit by reversing LVD and enhancing survival in patients already receiving ACE inhibitor treatment. For example, the MERIT and CIBIS-2 studies provide strong evidence of the benefits of metoprolol and bisoprolol. ${ }^{9}{ }^{10}$
Trial acronyms

AIRE: Acute Infarction Ramipril Efficacy

CIBIS-II: Cardiac Insufficiency Bisoprolol study-II

MERIT: Metroprolol Randomized Intervention Trial

RALES: Randomized Aldactone Evaluation Study

SAVE: Survival And Ventricular

Enlargement

SOLVD: Studies Of Left Ventricular Dysfunction

\section{Conclusion}

William Ostler said: "One should treat as many patients as possible with a new drug, while it still has the power to heal." There are now many therapeutic options for heart failure. It is therefore important that the evidence to support them is certain before they are used in patients. There is solid and convincing evidence that ACE inhibitors and $\beta$ blockers, and emerging evidence that aldosterone antagonism, can provide enhanced survival and new hope for the legions of patients worldwide who are afflicted with heart failure. There are other agents which show promise but which need further study before their introduction into clinical practice.

1 Kannel WB, Ho K, Thom T. Changing epidemiological features of cardiac failure. Br Heart f 1994;72 (suppl 2):S3-9.

2 Hammermeister KE, DeRouen TA, Dodge HT. Variables predictive of survival in patients with coronary disease. clinical, electrocardiographic, exercise, arteriographic, and quantitative evaluations. Circulation 1979;59:421-30.

3 Pitt D. ACE inhibitor co-therapy in patients with heart Pitt D. ACE inhibitor co-therapy in patients with heart
failure: rationale for the randomized aldactone evaluation study (RALES). Eur Heart f 1995;16(suppl N): 107-10.

4 Weber KT, Brilla CG. Pathological hypertrophy and cardiac interstitium. Fibrosis and renin-angiotensin-aldosterone system. Circulation 1991;83:1849-65.

5 Sharpe N, Murphy J, Smith H, et al. Treatment of patients with symptomless left ventricular dysfunction after myocardial infarction. Lancet 1988;i:255-9.

6 The SOLVD Investigators. Effect of enalapril on survival in patients with reduced left ventricular ejection fractions and congestive heart failure. N Engl 7 Med 1991;325:293-302.

7 Pfeffer MA, Braunwald E, Moye LA, et al. Effect of captopril on mortality and morbidity in patients with left ventricular dysfunction after myocardial infarction. $N \mathrm{Engl} f \mathrm{Med}$ 1992;327:669-77.

8 Lonn EM, Yusuf S, Jha P, et al. Emerging role of angiotensin-converting enzyme inhibitors in cardiac and vascular protection. Circulation 1994:90:2056-69.

9 MERIT-HF Investigators. Effect of metopolol CR/XL in chronic heart failure: metoprolol CR/XL randomised intervention trial in congestive heart failure (MERIT-HF). Lancet 1999;353:2001-7.

10 CIBIS-II Investigators. The cardiac insufficiency bisoprolol study II (CIBIS-II): a randomised trial. Lancet 1999;353:913. 